\title{
Advanced all-optical logic gates on a spectral bus
}

\author{
Per Olof Hedekvist, Ashish Bhardwaj, Kerry Vahala, and Henrik Andersson
}

\begin{abstract}
We present experimental results on a new method for ultrafast all-optical logic, which utilizes four-wave mixing on polarization-modulated signals. The technique allows advanced operations such as exclusive-OR and three-bit addition with carry bit. Furthermore, we show that on-the-fly errorcorrection encoding and decoding of a simple Hamming code is achieved when these gates are used on the bits of a spectrally structured word. These gates may be suitable for logic operations in an optoelectronic front end, which moves some of the necessary computation of data to the optical domain, before detection. (C) 2001 Optical Society of America

OCIS codes: $\quad 060.4510,190.4380,200.4660$.
\end{abstract}

\section{Introduction}

Because the bandwidth of optical fiber is much larger than the data rate of existing optoelectronic transmitters and receivers, signal multiplexing is used to utilize the fiber more efficiently. One of the most common techniques is wavelength division multiplexing (WDM), in which each transmitter uses an individual wavelength. In most present systems, the different multiplexed channels are uncorrelated, and data are transmitted serially. As an alternative to this, researchers at IBM suggested dispersing a binary word over the optical spectrum for transmission through a fiber (referred to here as a spectral bus) more than a decade ago. ${ }^{1}$ This spectral bus would correspond to a parallel data bus, which, however, is confined spatially within one fiber, with the different bits of each word on different WDM channels. Such a system would not only save space but also shorten the transmission time for a given data sequence. It was noted, however, that the performance of such systems was severely limited by fiberinduced group-velocity dispersion, which caused the different bits to travel at different velocities (also called bit skew). Since then, however, the idea of managing dispersion with one of several methods in-

When this research was performed the authors were with the Department of Applied Physics, MS 128-95, California Institute of Technology, Pasadena, California 91125. P. O. Hedekvist (poh@elm.chalmers.se) and H. Andersson are now with the Department of Microelectronics, Photonics Laboratory, Chalmers University of Technology, SE 412 96, Göteborg, Sweden.

Received 7 December 1999; revised manuscript received 5 December 2000.

0003-6935/01/111761-06\$15.00/0

(C) 2001 Optical Society of America cluding dispersion-compensating fiber, ${ }^{2}$ chirped fiber gratings ${ }^{3}$ or phase conjugation ${ }^{4}$ has become a practical reality. Consequently, the concept and potential attributes of a spectral bus should once again be revisited. Some recent results on the spectral bus in computer networks, ${ }^{5,6}$ and theoretical evaluations in local area networks, ${ }^{7}$ show the awakened interest in this transmission method.

Even though a simple spectral bus requires only a transmitter, a receiver, and some group-velocitydispersion compensation, it will not be fully utilized until some of the logic is performed in the optical domain. Possible applications include routing, encryption, and error detection-correction encoding, and decoding. In a previous work we proposed one possible realization of an all-optical spectral logic gate. ${ }^{8}$ This realization is ultrafast and has an internal architecture that permits programming of any two-input Boolean function.

Here we show experimental results on an alloptical front end, performing error correction on data that have been transmitted on a spectral bus. The unit is made from discrete components, as was the case in Ref. 8, but the unit can be partially integrated in a waveguide chip. Furthermore, the unit performs a three-input Boolean logic in one level, defined as the maximum number of sequential gates between the input and the output.

\section{Theory}

The all-optical logic operation of the device shown in this experiment is achieved by utilizing the nondegenerate four-wave mixing (FWM) process in a semiconductor optical amplifier (SOA). This process is an interaction between three input waves, and there will be an output wave only when there is energy in 

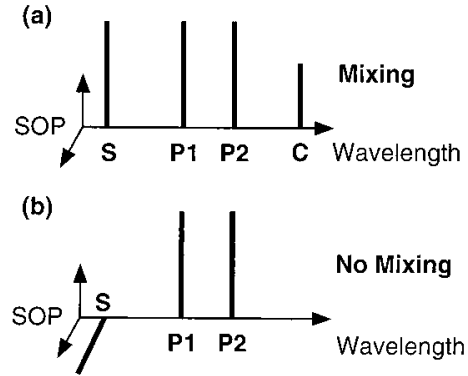

(c)

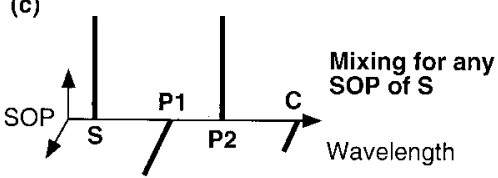

Fig. 1. Schematic diagram of the spectral characteristics of FWM. The three inputs, S, P1, and P2 corresponds to the signal, pump 1, and pump 2, respectively. The output $\mathrm{C}$ corresponds to the conjugate. (a) When all inputs are parallel, the efficiency is highest, and output is parallel to input. (b) When $\mathrm{S}$ is orthogonal to $\mathrm{P} 1$ and $\mathrm{P} 2$, there is no mixing. (c) When $\mathrm{P} 1$ is orthogonal to $\mathrm{P} 2$, then $\mathrm{C}$ will be orthogonal to $\mathrm{S}$.

all of them. Unfortunately, if the transmission fiber has low dispersion at the signal wavelengths, this process will undesirably take place in this region as well. Therefore a system utilizing a spectral bus must be based on another technique for minimizing group-velocity dispersion, such as one of the previously mentioned methods.

Even though several results have been presented on all-optical AND operation by use of FWM between intensity-modulated signals, ${ }^{9,10}$ an arbitrary all-optical logic gate requires a modulation method that permits interaction between both 1's and 0's. A wide-deviation frequency-shift keying method has previously been suggested to fulfill this requirement for a FWM-based device. ${ }^{11}$ As an alternative, we propose a data modulation that is based on binary polarization shift keying ${ }^{12}$ (PolSK), where the states of polarization (SOP) of the signal are orthogonal for 0 and 1 . In a system that uses polarizationmaintaining fiber, the SOP's are adjusted to coincide with the fast and the slow transmission axes in order for the original linear SOP's to be maintained during transmission. The bit skew induced by the different velocities for the 0's and the 1's can be compensated by $90^{\circ}$ cross splicing, forcing the fast and the slow axes to switch. Finally, at the receiver, the signal is transmitted through a polarizer, which changes the coding format to intensity modulated before detection. The polarization characteristics of interest in the FWM process between binary PolSK-modulated lightwaves are described graphically in Fig. 1. The new wave, $\mathrm{C}$, is created at the optical frequency $\omega_{\mathrm{c}}=$ $\omega_{\mathrm{P} 1}+\omega_{\mathrm{P} 2}-\omega_{\mathrm{S}}$, where $\omega_{\mathrm{P} 1}, \omega_{\mathrm{P} 2}$, and $\omega_{\mathrm{S}}$ are the optical frequencies of the three input waves $\mathrm{P} 1, \mathrm{P} 2$, and $\mathrm{S}$, respectively. When all input lightwaves are parallel [Fig. 1(a)] the mixing efficiency is the highest, and $\mathrm{C}$ has the same polarization as the inputs. Furthermore, when the signal is orthogonal to the two pump
Table 1. Polarization Mixing Table for First-Order Four-Wave Mixing

\begin{tabular}{cccc}
\hline $\mathrm{S}$ & $\mathrm{P} 1$ & $\mathrm{P} 2$ & $\mathrm{C}$ \\
\hline$\rightarrow$ & $\rightarrow$ & $\rightarrow$ & $\rightarrow$ \\
$\rightarrow$ & $\rightarrow$ & $\uparrow$ & $\uparrow$ \\
$\rightarrow$ & $\uparrow$ & $\vec{\uparrow}$ & $\uparrow$ \\
$\rightarrow$ & $\uparrow$ & $\uparrow$ & $\bullet$ \\
$\uparrow$ & $\rightarrow$ & $\rightarrow$ & $\rightarrow$ \\
$\uparrow$ & $\rightarrow$ & $\uparrow$ & $\vec{\bullet}$ \\
$\uparrow$ & $\uparrow$ & $\vec{\uparrow}$ & $\uparrow$ \\
\hline & $\uparrow$ & $\uparrow$ &
\end{tabular}

waves [Fig. 1(b)], there is no mixing. Finally, when the two pump waves are orthogonal and the signal is parallel to one of them [Fig. 1(c)], there will be mixing at half the efficiency of the case shown in Fig. 1(a), and the conjugate will be parallel to the other pump wave.

Mathematically, the output wave of the FWM process in a general nonlinear media can be described by ${ }^{13}$

$$
\begin{aligned}
E_{c} \hat{e}_{c}= & \eta E_{p 1} E_{p 2} E_{s\left[\mu_{p 1 s}\right.}^{*}\left(\hat{e}_{p 1} \cdot \hat{e}_{s}^{*}\right) \hat{e}_{p 2}+\mu_{p 2 s}\left(\hat{e}_{p 2} \cdot \hat{e}_{s}^{*}\right) \hat{e}_{p 1} \\
& \left.+\mu_{p 1 p 2}\left(\hat{e}_{p 1} \cdot \hat{e}_{p 2}\right) \hat{e}_{s}^{*}\right],
\end{aligned}
$$

where $E_{i}$ are the field amplitudes and $\hat{e}_{i}$ are the SOP vectors $(i=s, p 1, p 2, c$ corresponds to the signal, the two pumps, and the conjugate, respectively). Furthermore, $\eta$ is an efficiency coefficient, and $\mu_{i j}(i, j=$ $s, p 1, p 2)$ are mixing coefficients.

The last term in Eq. (1) corresponds to the mixing caused by a local interaction between the pump waves. In a rectangular waveguide this term can be neglected if the SOP vectors of the pumps coincide with either the TE or the TM mode. However, if the SOP's of the pump waves are not fully TE or TM, the last term is finite in a SOA. In this case the nonlinear effects depend on the input SOP even in a device in which the amplification is polarization independent. Therefore a reference axis is chosen parallel with the TM mode of the SOA. From Eq. (1) it is apparent that the output SOP is determined by a combination of the SOP's of the signal and both pump waves. Also, when the SOP's of the three lightwaves are orthogonal or parallel to the reference axis, the scalar products will be either 0 or 1 . Finally, $E_{c}=0$ only when $\hat{e}_{p 1}$ and $\hat{e}_{p 2}$ are parallel with each other and orthogonal to $\hat{e}_{s}$, as was described earlier in Fig. 1(b).

The described polarization properties are used to create a mixing table for nondegenerate FWM. In particular, in Table 1, the SOP of the conjugate, C, is shown for all possible cases of input SOP's that would occur when binary PolSK modulation is used. The notation used for the signal and pumps is the same as in Fig. 1: The arrows show vertical or horizontal polarization, and the small black dots correspond to the cases when no mixing occurs.

To fully utilize the polarization properties of FWM for spectral logic, the process must be nondegenerate, i.e., all light waves are at different wavelengths. However, FWM will also occur if the two pump waves 
coincide $\left(\omega_{\mathrm{P} 1}=\omega_{\mathrm{P} 2}\right)$ in a partially degenerate FWM process. Such a process was used, e.g., in Ref. 8 to perform exclusive-OR (XOR) between two signals. Hence when three light waves are launched through the nonlinear media, six partially degenerate FWM processes will occur simultaneously with three nondegenerate processes. This is why some effort has to be put into filtering out the correct conjugated signal with high suppression of the adjacent spurious mixing products.

\section{A. Three-Bit Logic Gates}

We have utilized the polarization properties of nondegenerate FWM to perform advanced all-optical logic functions, with as many as three inputs. These logic units are based on the mixing properties shown in Table 1, and some general comments are in order. A fully functional logic gate must have a specified output for each combination of inputs, and because there are cases in Table 1 in which no mixing occurs, more than one SOA may be necessary. In the gate they are situated parallel to each other, and to avoid interference effects, mixing must occur in only one SOA for each case of the truth table. To achieve this, it may be necessary to insert polarizers to reduce the cases of mixing in Table 1 to a single case: all parallel with the polarizer. Furthermore, to fill the blanks, the signals must undergo some pre-SOA processing. This processing could be, e.g., a half-wavelength shift, which corresponds to inversion of PolSK-modulated signals. It can also be a wavelength-selective halfwavelength shift, which changes the SOP of only one of the lightwaves, while leaving the SOP's of the remaining two waves unaffected. Furthermore, the pre-SOA processing could be made with or without a polarizer, limiting the number of cases covered to one or six, respectively.

Figure 2 shows the design of a gate by use of three parallel SOA's. The arrows in the tables show the possible combinations of SOP's at each stage. For the binary PolSK-modulated data, the convention used here is that a horizontal arrow corresponds to 0 and a vertical arrow corresponds to 1 . In the SOA's the first data bit, D1, corresponds to the signal wave, $\mathrm{S}$, and the two other data bits, D2 and D3, correspond to the two pump waves, $\mathrm{P} 1$ and $\mathrm{P} 2$, respectively. The pre-SOA processing in the upper arm (SOA1) is performed by a birefringent element $(\mathrm{BE})$ that inverts the SOP of $\mathrm{P} 1$ and $\mathrm{P} 2$ but not the SOP of S. In the middle and lower arms the pre-SOA processing consists of polarizers (arrows), mounted vertically (before SOA2) and horizontally (before SOA3). Each of the four-column tables shows the SOP's of the signal, pumps and conjugate $(\mathrm{C})$ of the FWM process in each SOA, after the pre-SOA processing. The black dots correspond to no power in $\mathrm{C}$, and because each row consists only of power from one SOA, the output (OUT) can be achieved through combining the conjugates of the three arms. Together with the three input columns [D1, D2, D3] to the left in Fig. 2, the OUT column to the right fulfills the truth table, and it can be noted that this is the output bit of a three-bit

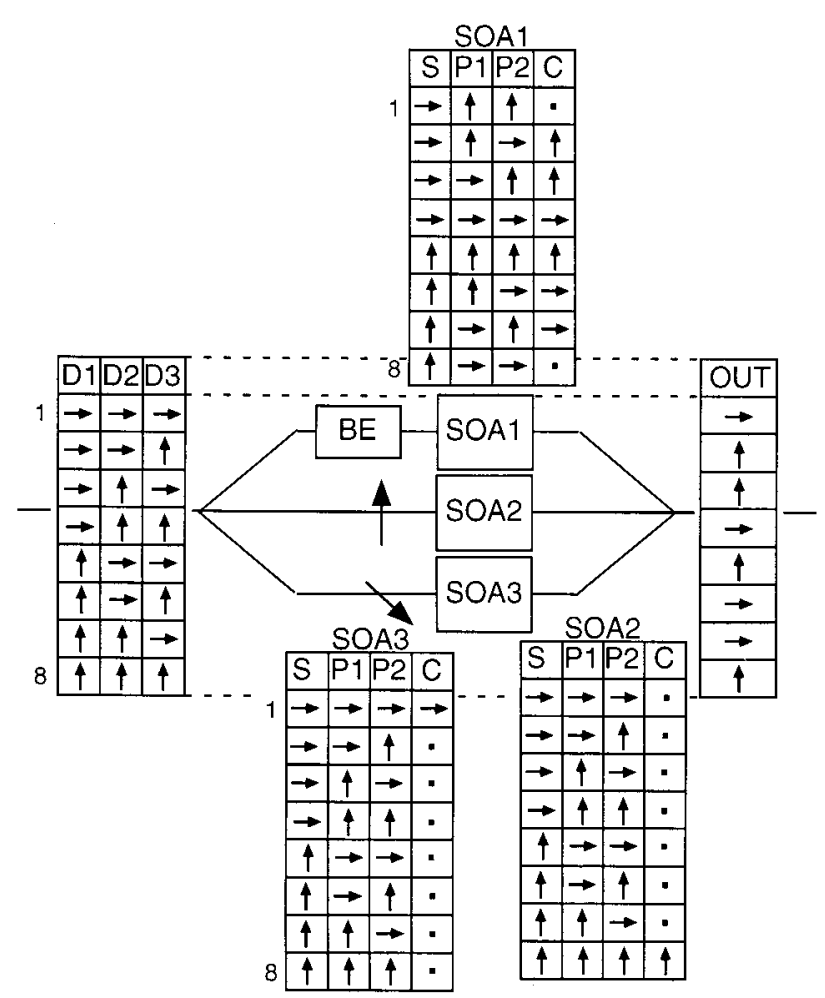

Fig. 2. Design of an all-optical three-bit adder by use of FWM between PolSK-modulated signals in three parallel SOA's.

modulo-2 addition. In this design for logic opertions, the different input cases in the truth table are treated in three different SOA's, with each case tested by only one SOA.

The necessity of parallel devices requires synchronization at the time scale of the data rate, but this is not a serious limitation. If the device were integrated on a waveguide chip, sub-ps accuracy could easily be achieved.

\section{B. Error-Correction Front End}

To permit detection and correction of errors in transmission on an optical fiber, there must be an addition of parity bits. Currently, this encoding and decoding is performed in the electrical domain, before transmission and after detection, respectively. However, in a simple fiber-optic data bus that uses PolSK-modulated byte-wide transmission, the errorcorrection (EC) coding can be implemented with alloptical spectral logic. In such a system the EC can be obtained in the optical receiving front end, as the final step before detection.

The EC code we have used is the simplest for single-error correction, the Hamming $(3,1)$, which uses one data bit and two parity bits. The transmitted word is then either $[D 1, D 2, D 3]=[0,0,0]$ or $[1,1,1]$. In this case each bit, $D i$, corresponds to a different wavelength, $\lambda_{i}(i=1,2,3)$. If an error occurs on either bit, an undesired word is received. With the EC receiver, the erratic bit is detected, and the output data corrected. Because the EC device is 
Table 2. Truth Table for Decoding the Hamming $(3,1)$ Code

\begin{tabular}{cccc}
\hline D1 & D2 & D3 & OUT \\
\hline 0 & 0 & 0 & 0 \\
0 & 0 & 1 & 0 \\
0 & 1 & 0 & 0 \\
0 & 1 & 1 & 1 \\
1 & 0 & 0 & 0 \\
1 & 0 & 1 & 1 \\
1 & 1 & 0 & 1 \\
1 & 1 & 1 & 1 \\
\hline
\end{tabular}

a part of the receiver, only the data bit must be recovered, while the parity bits have been used and can be left aside. Even though this is a rudimentary code, with a high redundancy, it nonetheless demonstrates the feasability of all-optical front-end error correction.

The truth table for the EC device is shown in Table 2. If there are two or three 1's at the input, the output should also be 1 ; otherwise, it should be 0 . This operation is also recognized as the carry bit of a three-bit addition. It gives a logical expression that can be written as

$$
\mathrm{OUT}=(D 1 \wedge D 2) \vee(D 1 \wedge D 3) \vee(D 2 \wedge D 3),
$$

where $\wedge$ is the logical AND and $\vee$ is the logical oR. This expression can be realized in a circuit made from fundamental two-input logic gates, and one possible design is shown in Fig. 3. However, it should be noted that this requires three levels of gates. In contrast, in the all-optical domain the full truth table can be achieved in one level. With binary PolSK modulation, horizontal polarization corresponds to 0 and vertical polarization to 1 . Use of the outcome of nondegenerate FWM in one SOA (Table 1), and by letting D1 correspond to the inverse of S, and D2 and D3 correspond to $\mathrm{P} 1$ and $\mathrm{P} 2$, respectively, the six middle cases are achieved. This pre-SOA processing is similar to the example in Fig. 2, but with the opposite selection of which wavelengths to invert. Now there will be no mixing only when all three inputs are alike, which again are the cases that must be treated in separate SOA's, connected in parallel with the first. A vertically aligned polarizer in front

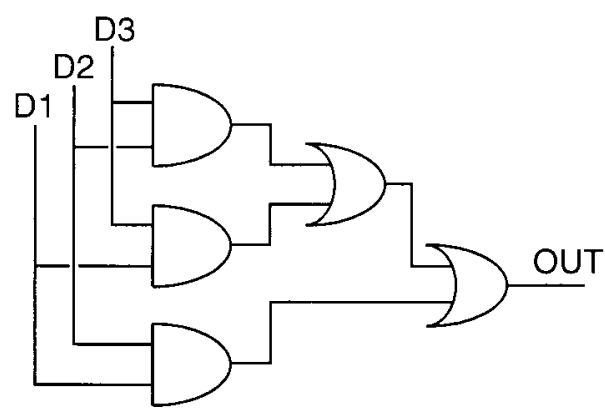

Fig. 3. Logic circuit for three-bit error correction, using the fundamental AND and oR gates.

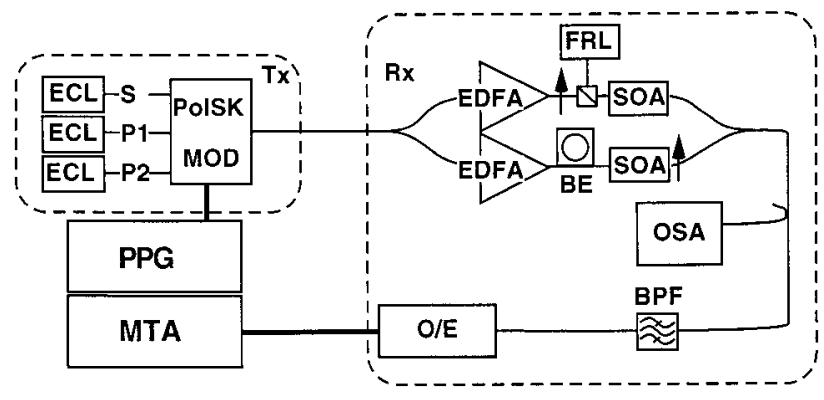

Fig. 4. Experimental setup for testing the Hamming $(3,1)$ EC device.

of one SOA will limit the mixing to the case of $[1,1,1]$, and a horizontally aligned polarizer before another SOA will mix the case of $[0,0,0]$. Finally, the birefringence axes of the outcoupling fibers must be properly aligned, to preserve the PolSK coding.

\section{Experiment}

For increased stability and owing to the inherent polarization dependence in the SOA's, the experiment is performed solely in polarization-maintaining fiber, and the setup is shown in Fig. 4. The transmitter (Tx) consists of three tunable external cavity lasers (ECL), used as signal sources. The signal wavelength (S) was $1549.9 \mathrm{~nm}$, and the pump wavelengths were $1547.8 \mathrm{~nm}(\mathrm{P} 1)$ and $1546.8 \mathrm{~nm}(\mathrm{P} 2)$. All three signals are PolSK-modulated at $2.5 \mathrm{Gbit} / \mathrm{s}$ with a bit sequence generated in the pseudo-random pattern generator (PPG). The modulator allows any one wavelength to be altered independently of the other two, and in the WDM coding, D1, D2 and D3 were assigned to S, P1 and $\mathrm{P} 2$, respectively. At the receiver $(\mathrm{Rx})$, the light is divided into two equal parts, each amplified in an erbium-doped fiber amplifier (EDFA) and launched into a SOA. The arrows indicate the positions of polarizers that convert PolSK modulation into intensity modulation. In the upper arm, there will only be mixing when the input word is $[1,1,1]$. All other cases will mix in the lower arm, at which the pre-SOA processing consists of a BE, which changes the SOP of $S$ by $90 \mathrm{deg}$ while leaving the SOP's of P1 and P2 essentially unaffected. The BE consist of a 75-cm-long polarization-maintaining fiber, with its birefringent axis spliced at a $45^{\circ}$ angle to the axis of the input and output fibers. Thus the wavelength difference for which the fiber works as a half-wave plate and a fullwave plate is approximately $2.5 \mathrm{~nm}$ for the fiber used in the experiment. Furthermore, the temperature of the fiber is adjustable to permit the tunability and increased stability of the wavelength selection.

Finally, the two arms are combined, and the conjugate is filtered out. Any interference between the outputs of the two arms is minimized because there is never mixing in both SOA's simultaneously. Furthermore, since the modulation of the signal is changed to intensity modulated after the gate, the SOA that should mix the case of $[0,0,0]$, emitting 0 , can and has been omitted. The FWM process is ob- 


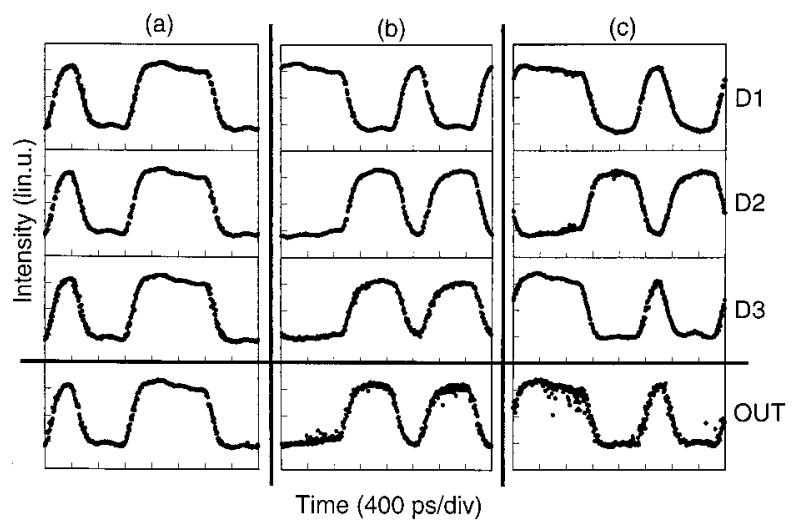

Fig. 5. Input and output sequences of the EC device when there are (a) no errors, (b) errors in D1, and (c) errors in D2.

served in the frequency domain using an optical spectrum analyzer (OSA), and data is filtered out in an optical bandpass filter (BPF) and detected with an optically preamplified optoelectronic converter $(\mathrm{O} / \mathrm{E})$. The sequences in the time domain are observed with the microwave transition analyzer (MTA).

In full operation the optical power levels of the signal and pumps, launched into the SOA's, are high (10-100 $\mathrm{mW})$, and the SOA's are heavily saturated. However, when the error-free $[0,0,0]$ case is received, no optical power will be launched through the upper SOA, so that it quickly becomes unsaturated. In this case there would be substantial cross-gain modulation and noise addition unless an additional wave is included, which constantly limits the gain of the SOA. This idler wave is emitted from a fiber-ring laser (FRL) at lower power and at a higher wavelength $(1560 \mathrm{~nm})$ and combined with the data signals at orthogonal polarization. Thus this wave will not interfere with the FWM process by inducing any spurious waves in the vicinity of the output wave.

The functionality of the gate is shown in Fig. 5, where the three input bits, D1-D3 and the output bit, OUT, are arranged vertically. If there are no errors at the transmitter, the output bit is the same as the inputs, as seen in Fig. 5(a). Furthermore, if either D1 or D2 is erroneous, the correct data are still recovered, as shown in Figs. 5(b) and 5(c), respectively. The case of errors in D3 is not shown because it corresponds to the identical mixing process as errors in D2. The noise in the output in Fig. 5(c) indicates that this is the combination with the lowest FWM efficiency. Nevertheless, the measurements show that the logic function of the gate performs as expected.

Figure 6 shows the spectra when $[1,1,1]$ is launched into the gate. The solid curve shows the spectrum at the output of the upper SOA, where the mixing occurs, and the dotted curve shows the spectrum at the output of the lower SOA, where the FWM is minimized. The spectra are expanded in the vicinity of the output signal, and it shows that the suppression between the outputs is close to $20 \mathrm{~dB}$. Furthermore,

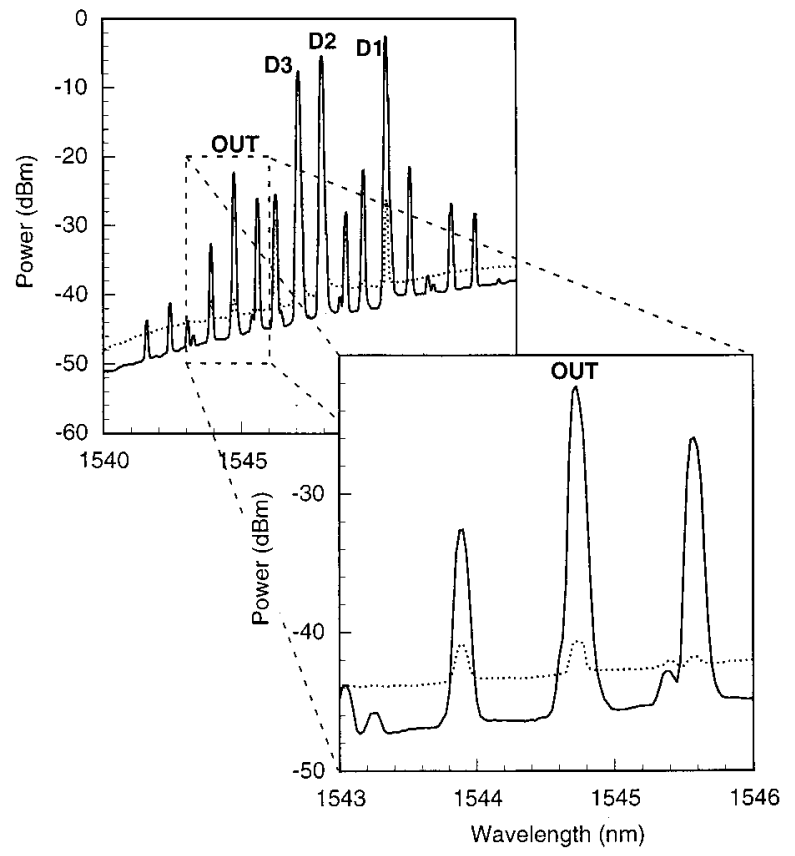

Fig. 6. Spectra at the output of upper SOA (solid curve) and lower SOA (dotted curve) when $[1,1,1]$ is launched into the EC gate. The area close to the output signal (OUT) is expanded for clarity.

all the adjacent peaks arise from other FWM processes within the SOA, and they must be suppressed before detection.

\section{Conclusion}

We propose a new technique for advanced all-optical logic on a spectral bus, transmitting data on bytewide WDM channels. The technique utilizes FWM of PolSK-modulated signals and permits three-input logic functions in one level of SOA gates. These gates show that advanced all-optical logic functions can be achieved without use of the basic building blocks of electronics, such as NAND and NOR.

Furthermore, we have shown experimentally the function of an all-optical error-correcting device, decoding the Hamming $(3,1)$ code. The device is characterized dynamically at $2.5 \mathrm{Gbit} / \mathrm{s}$, with all input signals modulated and both with and without errors added. This is, to the best of our knowledge, the first three-input all-optical logic gate, performed in one level, that has been experimentally verified.

We have also described how an all-optical three-bit adder with carry bit, operating on PolSK-modulated signals, can be built from six parallel SOA's. This shows the flexibility of the use of polarization properties of FWM, as well as the complexity of gates that can be realized.

It is important to emphasize that the bit rates used in this experiment are limited only by the electronic circuitry of the test instruments - not by the spectral logic. Optical signal processing by FWM in a SOA has been demonstrated at bit rates to as much as $40 \mathrm{Gbit} / \mathrm{s}$, and none of the other (passive) components in this experiment pose an intrinsic limit to the bit 
rate of the individual signals. ${ }^{14}$ In addition, the wavelength-multiplexed nature of the gates makes the extension to larger aggregate bit rates straightforward. Furthermore, because the nonlinear elements of the device are commercially available SOA's, the input power levels must be of the order of $100 \mathrm{~mW}$. To keep the power level low during transmission, amplifiers are needed or a material with higher nonlinearity must be used. The proposal for a spectral bus originates from $1985^{1}$ as a method of saving space and material, in comparison with electrical ribbon cables. Another benefit is the capabilities of all-optical ultrafast logic operations between the data bits, similar to the gates described in this paper. We believe that the spectral bus is advantageous, not only over a parallel electrical data bus but also over a parallel optical data bus that utilizes a fiber bundle with one single-bit transmission in each. In the latter, the electronics is merely replaced by optics, but there is less possibility to utilize fully the potential offered by all-optical processing.

This research is supported by the Ballistic Missile Defense Organization and the Air Force Office of Scientific Research AFOSR grants F49620-97-1-0512 and F49620-98-1-0409. P. O. Hedekvist is supported by the Swedish Foundation for International Cooperation in Research and Higher Education (STINT).

\section{References}

1. M. L. Loeb and G. R. Stilwell, Jr., "High-speed data transmission on an optical fiber using a byte-wide WDM system," J. Lightwave Technol. 6, 1306-1311 (1988).

2. R. Nuyts, Y. Park, and P. Gallion, "Dispersion equalization of a $10 \mathrm{~Gb} / \mathrm{s}$ repeatered transmission system using dispersion compensating fibers," J. Lightwave Technol. 15, 31-42 (1997).

3. K. Hill, F. Bilodeau, B. Malo, T. Kitagawa, S. Theriault, D.
Johnson, and J. Albert, "Chirped in-fiber Bragg gratings for compensation of optical fiber dispersion," Opt. Lett. 19, 13141316 (1994).

4. A. Yariv, D. Fekete, and D. Pepper, "Compensation for channel dispersion by nonlinear optical phase conjugation," Opt. Lett. 4, 52-54 (1979).

5. L. A. Bergman, A. J. Mendez, and L. S. Lome, "Bit-parallel links for high-performance computer networks," in Optoelectronic Interconnects and Packaging, R. T. Chen and P. S. Guilfoyle, eds., Proc. SPIE CR62, 210-226 (1996).

6. L. Bergman, J. Morookian, and C. Yeh, "An all-optical longdistance multi-Gbyte/s bit-parallel WDM single-fiber link," J. Lightwave Technol. 16, 1577-1582 (1998).

7. C. C. Chen, L. A. Wang, and S.-Y. Kuo, "A wavelength encoded multichannel optical bus for local area networks," J. Lightwave Technol. 14, 315-323 (1996).

8. K. Vahala, R. Paiella, and Guido Hunziker, "Ultrafast WDM logic," IEEE J. Sel. Top. Quantum Electron. 3, 698-701 (1997).

9. D. Nesset, M. C. Tatham, L. D. Westbrook, and D. Cotter, "Degenerate wavelength operation of an ultrafast all-optical AND gate using four-wave mixing in a semiconductor optical amplifier," Electron. Lett. 30, 1938-1940 (1994).

10. T. Morioka, H. Takara, S. Kawanishi, K. Uchiyama, and M. Saruwatari, "Polarization-independent all-optical demultiplexing up to $200 \mathrm{Gbit} / \mathrm{s}$ using four-wave mixing in a semiconductor optical amplifier," Electron. Lett. 32, 840-842 (1996).

11. A. D'Ottavi, E. Iannone, and S. Scotti, "Address recognition in all-optical packet switching by fwm in semiconductor amplifier," Microwave Opt. Technol. Lett. 10, 228-230 (1995).

12. S. Benedetto, R. Gaudino, and P. Poggiolini, "Direct detection of optical digital transmission based on polarization shift keying modulation," IEEE J. Sel. Areas Commun. 13, 531-542 (1995).

13. B. Ya. Zel-dovich, N. F. Pilipetsky, and V. V. Shkunov, Principles of Phase Conjugation (Springer-Verlag, Berlin, 1985), pp. 150-152.

14. D. Nesset, D. D. Marcenac, P. L. Mason, A. E. Kelly, S. Bouchoule, and E. Lach, "Simultaneous wavelength conversion of two $40 \mathrm{Gbit} / \mathrm{s}$ channels using four-wave mixing in a semiconductor optical amplifier,” Electron. Lett. 34, 107-108 (1998). 